\title{
Eigenvalue instantons in the spectral form factor of random matrix model
}

\author{
Kazumi Okuyama \\ Department of Physics, Shinshu University, \\ 3-1-1 Asahi, Matsumoto, Nagano 390-8621, Japan \\ E-mail: kazumi@azusa.shinshu-u.ac.jp
}

ABstract: We study the late time plateau behavior of the spectral form factor in the Gaussian Unitary Ensemble (GUE) random matrix model. The time derivative of the spectral form factor in the plateau regime is not strictly zero, but non-zero due to a nonperturbative correction in the $1 / N$ expansion. We argue that such a non-perturbative correction comes from the eigenvalue instanton of random matrix model and we explicitly compute the instanton correction as a function of time.

KEYwords: 1/N Expansion, Matrix Models, Random Systems

ARXiv EPrint: 1812.09469 


\section{Contents}

1 Introduction $\quad 1$

2 Exact spectral form factor at $\beta=0 \quad 2$

$\begin{array}{lll}3 & \text { Eigenvalue instanton in the spectral form factor } & 6\end{array}$

$\begin{array}{lll}4 & 1 / N \text { expansion around the instanton } & 8\end{array}$

5 Comment on the $\beta \neq 0$ case 9

6 Conclusion and outlook $\quad 11$

\section{Introduction}

The spectral form factor (SFF) is first introduced in [1] as a Fourier transform of the twolevel correlation function of energy eigenvalues. It is a very basic and important quantity in the study of quantum chaos; it is widely used in many areas of physics ranging from nuclear physics to condensed matter physics, to name a few, and even the application to quantum gravity and black holes is discussed in the literature [2]. At late times, SFF of a chaotic system exhibits a universal behavior, so-called ramp and plateau, and it is described by a random matrix theory of a certain ensemble of matrices depending on the symmetry of the system.

As an application to quantum gravity, the SFF in the Sachdev-Ye-Kitaev (SYK) model [3-5] has been intensively studied as a useful diagnostic of the chaotic behavior of the dual black hole in two dimensions [2]. It is observed numerically that the SFF of the SYK model indeed exhibits the structure of ramp and plateau as expected for a chaotic system. In the recent paper [6], the structure of ramp was studied from the viewpoint of the collective field theory in terms of the variables $G, \Sigma$, and the possible interpretation of the bulk geometry was discussed. However, the structure of plateau in the SYK model is not yet fully understood. It is speculated that the plateau in the SYK model is closely related to the non-perturbative effects in the large $N$ expansion $[2,6]$.

Motivated by this conjecture in the SYK model, in this paper we will consider a much simpler example of the plateau in the Gaussian Unitary Ensemble (GUE) random matrix model. The SFF in the GUE random matrix model was extensively studied in the literature and the exact results in the large $N$ limit as well as the exact results at finite $N$ are available (see e.g. [7-9]). In the plateau regime, the SFF is approximately constant in time and its time derivative vanishes in the large $N$ limit. However, at finite $N$ it is non-zero due to a non-perturbative effect in the $1 / N$ expansion. 
We will argue that such a non-perturbative correction comes from the eigenvalue instanton in the matrix integral, where some of the eigenvalues are pulled out from the cut and put on the non-trivial saddle points of the eigenvalue integral $[10,11] .{ }^{1}$ Naively, one might think that there is no non-perturbative correction in the $1 / N$ expansion of Gaussian matrix model. However, we should recall that by the "late time" we mean the following scaling limit

$$
N \rightarrow \infty, t \rightarrow \infty, \quad \text { with } \tau=\frac{t}{2 N} \text { fixed. }
$$

Since the SFF is defined by inserting the operator $\operatorname{Tr} e^{ \pm \mathrm{i} t H}=\operatorname{Tr} e^{ \pm 2 \mathrm{i} N \tau H}$ into the matrix integral, there appears a non-trivial saddle point in the scaling limit (1.1) due to the $\mathcal{O}(N)$ potential exerted on the eigenvalues. It turns out that the saddle points are located on the imaginary axis since the exponent of $\operatorname{Tr} e^{ \pm 2 \mathrm{i} N \tau H}$ is pure imaginary.

Interestingly, the computation of the non-perturbative correction to the SFF in the plateau regime is formally equivalent to the similar computation in the 2d Yang-Mills theory on $S^{2}$ at weak coupling. In fact, the one-point function of the operator $\operatorname{Tr} e^{ \pm 2 \mathrm{i} N \tau H}$ in the GUE matrix model, whose exact form at finite $N$ is given by the Laguerre polynomial (2.6), is exactly the same as the expression appeared in the study of the partition function of $2 \mathrm{~d}$ Yang-Mills theory on $S^{2}$ [13] under a certain identification of the parameters. This implies that, at least technically, the ramp-plateau transition in the SFF of the GUE random matrix model is related to the Douglas-Kazakov phase transition of the 2d Yang-Mills theory on $S^{2}[14]$.

This paper is organized as follows. In section 2, after reviewing the exact result of the $\mathrm{SFF}$ in the GUE random matrix model, we consider the non-perturbative correction to the SFF using the formal relation to the non-perturbative correction in the 2d Yang-Mills theory on $S^{2}$. In section 3, we argue that the non-perturbative correction to the SFF comes from the eigenvalue instantons. In section 4 , we compute the $1 / N$ corrections around the instanton configuration using the differential equation obeyed by the Laguerre polynomials. In section 5 , we briefly comment on the case of the non-zero inverse temperature $\beta \neq 0$. Finally, we conclude in section 6 .

\section{Exact spectral form factor at $\beta=0$}

Let us first review the exact form of the SFF in the GUE random matrix model at finite $N$ obtained in [9]. In the following, we will set the inverse temperature $\beta=0$ for simplicity. We will comment on the $\beta \neq 0$ case in section 5 .

The SFF in the GUE random matrix model is defined by

$$
g(\tau)=\left\langle\operatorname{Tr} e^{\mathrm{i} t H} \operatorname{Tr} e^{-\mathrm{i} t H}\right\rangle,
$$

where the expectation value is given by the Gaussian integral over the $N \times N$ hermitian matrix $H$

$$
\langle\cdots\rangle=\frac{\int d H e^{-\frac{N}{2} \operatorname{Tr} H^{2}}(\cdots)}{\int d H e^{-\frac{N}{2} \operatorname{Tr} H^{2}}} .
$$

\footnotetext{
${ }^{1}$ A collective field approach to the non-perturbative corrections to the SFF was studied by Andreev and Altshuler in [12]. We will not consider that approach in this paper.
} 
We are interested in the late time behavior of the SFF in the scaling limit (1.1), and we use $\tau=t / 2 N$ as the time coordinate in what follows. The SFF is naturally decomposed into the disconnected part $g_{\text {disc }}(\tau)$ and the connected part $g_{\text {conn }}(\tau)$

$$
g(\tau)=g_{\text {disc }}(\tau)+g_{\text {conn }}(\tau)
$$

The disconnected part is given by the product of one-point functions

$$
g_{\text {disc }}(\tau)=\mathcal{Z}(\tau)^{2}
$$

with $\mathcal{Z}(\tau)$ being the one-point function

$$
\mathcal{Z}(\tau)=\left\langle\operatorname{Tr} e^{\mathrm{i} t H}\right\rangle=\left\langle\operatorname{Tr} e^{-\mathrm{i} t H}\right\rangle .
$$

Note that $\mathcal{Z}(\tau)$ is an even function of $\tau$ and it does not dependent on the sign of $\tau$. The exact form of $\mathcal{Z}(\tau)$ at finite $N$ is given by

$$
\mathcal{Z}(\tau)=e^{-2 N \tau^{2}} L_{N-1}^{1}\left(4 N \tau^{2}\right)
$$

where $L_{n}^{\alpha}(x)$ denotes the associated Laguerre polynomial. One can also write down the exact form of the connected part $g_{\text {conn }}(\tau)$ as a trace of some $N \times N$ matrix [9]. It turns out that the time derivative of the connected part $\partial_{\tau} g_{\text {conn }}(\tau)$ has a simple expression [9]

$$
\frac{\partial g_{\mathrm{conn}}(\tau)}{\partial \tau}=8 N^{2} \tau e^{-4 N \tau^{2}}\left[L_{N-1}\left(4 N \tau^{2}\right) L_{N-1}^{1}\left(4 N \tau^{2}\right)-L_{N}\left(4 N \tau^{2}\right) L_{N-2}^{1}\left(4 N \tau^{2}\right)\right] .
$$

Moreover, using the property of Laguerre polynomials, one can show that the connected part $g_{\text {conn }}(\tau)$ and the disconnected part $g_{\text {disc }}(\tau)$ are related by $[7]$

$$
g_{\mathrm{disc}}(\tau)=-\frac{1}{8 N \tau} \frac{\partial}{\partial \tau}\left(\frac{1}{8 N \tau} \frac{\partial g_{\mathrm{conn}}(\tau)}{\partial \tau}\right)=-\frac{\partial^{2} g_{\mathrm{conn}}(\tau)}{\partial a^{2}}, \quad\left(a=4 N \tau^{2}\right)
$$

We emphasize that this relation holds exactly at finite $N$.

In the large $N$ scaling limit $(1.1), \partial_{\tau} g_{\text {conn }}(\tau)$ behaves differently below and above the critical time $\tau=1[9]$

$$
\partial_{\tau} g_{\mathrm{conn}}(\tau)= \begin{cases}\frac{4 N}{\pi} \sqrt{1-\tau^{2}}, & (\tau<1) \\ 0, & (\tau>1)\end{cases}
$$

Namely, when $\tau<1 \partial_{\tau} g_{\text {conn }}(\tau)$ obeys the semi-circle law as a function of time [9], while $\partial_{\tau} g_{\text {conn }}(\tau)$ vanishes beyond $\tau=1$. The regime $\tau<1$ corresponds to the ramp and the regime $\tau>1$ corresponds to the plateau. In the rest of this paper, we will consider the behavior of the SFF in the plateau regime $\tau>1$.

The vanishing of $\partial_{\tau} g_{\text {conn }}(\tau)$ in the plateau regime $\tau>1$ in (2.9) is a result of the strict large $N$ limit. At finite $N, \partial_{\tau} g_{\text {conn }}(\tau)$ is non-zero but exponentially suppressed when $N \gg 1$. It turns out that this exponentially small correction is non-perturbative in the $1 / N$ expansion, and the study of such non-perturbative corrections is the main purpose of this 
paper. Using the relations $(2.4)$ and (2.8), the non-perturbative correction to $\partial_{\tau} g_{\text {conn }}(\tau)$ can be obtained once we know the non-perturbative correction to the one-point function $\mathcal{Z}(\tau)$. As we will see shortly, $\mathcal{Z}(\tau)$ in the plateau regime behaves as

$$
\mathcal{Z}(\tau) \sim e^{-N S_{\text {inst }}(\tau)},
$$

with the "instanton action" $S_{\text {inst }}(\tau)$ being

$$
S_{\text {inst }}(\tau)=2\left[\tau \sqrt{\tau^{2}-1}-\operatorname{arccosh}(\tau)\right] .
$$

From the relations $(2.4)$ and $(2.8)$ it follows that $\partial_{\tau} g_{\text {conn }}(\tau)$ receives the two-instanton correction

$$
\partial_{\tau} g_{\mathrm{conn}}(\tau) \sim e^{-2 N S_{\mathrm{inst}}(\tau)} .
$$

Let us consider the large $N$ behavior of $\mathcal{Z}(\tau)$ in (2.6) in the plateau regime. Interestingly, the asymptotic behavior of the Laguerre polynomial in (2.6) has been studied in a different context in [13]; exactly the same expression as (2.6) appeared in the study of the partition function of 2d Yang-Mills theory on $S^{2}$ with the identification

$$
\tau \leftrightarrow \frac{\pi^{2}}{A},
$$

where $A$ is the 't Hooft coupling of $2 \mathrm{~d}$ Yang-Mills theory. With this identification, the plateau regime $\tau>1$ corresponds to the weak coupling phase $A<\pi^{2}$ of 2 d Yang-Mills theory and the transition at $\tau=1$ from the ramp to the plateau corresponds to the Douglas-Kazakov phase transition of 2d Yang-Mills theory on $S^{2}$ [14]. The "instanton action" $\gamma\left(A / \pi^{2}\right)$ in the 2d Yang-Mills theory has been computed in [13] using the integral representation of the Laguerre polynomial and it can be translated to the instanton action $S_{\text {inst }}(\tau)$ in $(2.11)^{2}$ via the dictionary $(2.13)$.

We can numerically check the expected behavior of $\partial_{\tau} g_{\text {conn }}(\tau)$ in $(2.12)$ using the exact form of $\partial_{\tau} g_{\text {conn }}(\tau)$ in $(2.7)$. One can extract the instanton action from the exact result of $\partial_{\tau} g_{\text {conn }}(\tau)$ in $(2.7)$ as

$$
S_{\mathrm{inst}}(\tau) \approx-\frac{1}{2 N} \log \left(\partial_{\tau} g_{\mathrm{conn}}(\tau)\right), \quad(N \gg 1) .
$$

In figure 1, we plot the exact value of the right hand side of (2.14) for $N=500$ in the plateau regime $\tau>1$. One can see from figure 1 that the exact result of $\partial_{\tau} g_{\text {conn }}(\tau)$ in $(2.7)$ correctly reproduces the analytic form of the instanton action in (2.11) as expected.

Note that the instanton action $S_{\text {inst }}(\tau)$ in (2.11) vanishes at the critical time $\tau=1$. In the context of $2 \mathrm{~d}$ Yang-Mills theory on $S^{2}$, this vanishing behavior of instanton action is interpreted in [13] that the Douglas-Kazakov phase transition is induced by the instantons. In the case of the SFF, it is tempting to speculate that the ramp-plateau transition is also induced by the instantons. It would be extremely interesting to study this picture in the

\footnotetext{
${ }^{2}$ Interestingly, a similar expression has also appeared in the instanton computation of the Gross-WittenWadia unitary matrix model (see e.g. eq. (4.23) in [15]).
} 


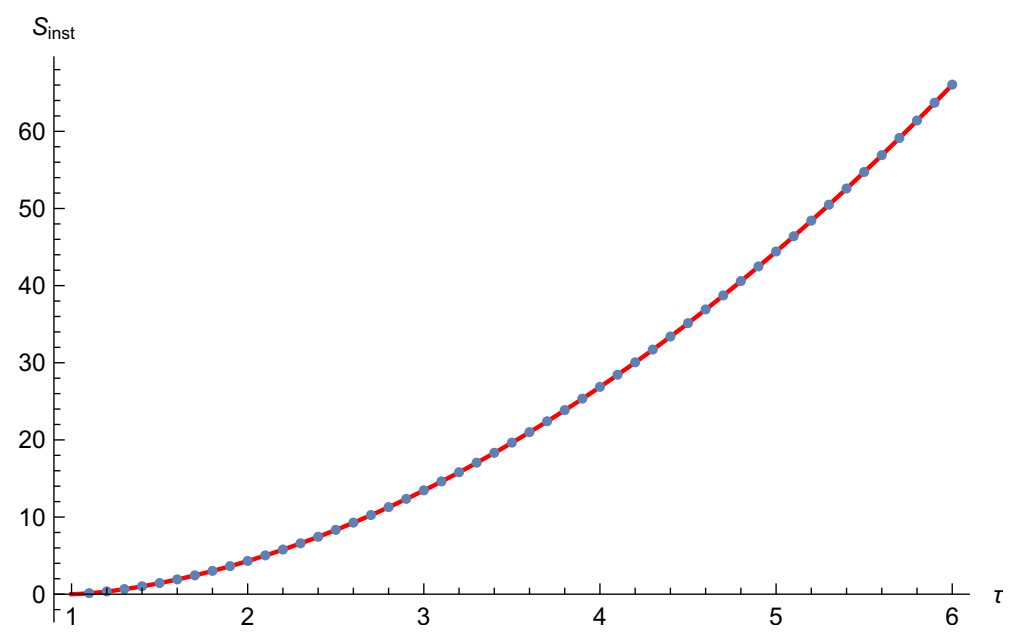

Figure 1. Plot of the instanton action $S_{\text {inst }}(\tau)$. The dots are the exact values of the right hand side of (2.14) for $N=500$, while the red curve represents the analytic form of $S_{\text {inst }}(\tau)$ in $(2.11)$.

holographic dual of the SYK model, called JT string in [6], and see how the D-branes in the JT string are related to the ramp-plateau transition.

We also notice that the expression of $\mathcal{Z}(\tau)$ in (2.6) has another interesting interpretation in the $4 \mathrm{~d} \mathcal{N}=4$ super Yang-Mills (SYM) theory. The expectation value of $1 / 2$ BPS Wilson loop $W_{k}$ in $4 \mathrm{~d} \mathcal{N}=4$ SYM theory with winding number $k$ is given by $[16,17]^{3}$

$$
\left\langle W_{k}\right\rangle_{\mathcal{N}=4 \mathrm{SYM}}=\frac{1}{N} e^{2 N \kappa^{2}} L_{N-1}^{1}\left(-4 N \kappa^{2}\right),
$$

where $\kappa$ is given by

$$
\kappa=\frac{k \sqrt{\lambda}}{4 N}
$$

and $\lambda$ denotes the 't Hooft coupling of $\mathcal{N}=4$ SYM. As discussed in [17], in the limit of large winding number $k \sim \mathcal{O}(N)$

$$
k \rightarrow \infty, N \rightarrow \infty \text {, with } \kappa \text { fixed, }
$$

the holographically dual object of the winding Wilson loop becomes a D3-brane embedded in $A d S_{5} \times S^{5}$ and $\kappa$ corresponds to the electric flux on the D3-brane worldvolume. One can see that the Wilson loop in $\mathcal{N}=4 \mathrm{SYM}$ in $(2.15)$ and $\mathcal{Z}(\tau)$ in (2.6) are related by an analytic continuation

$$
\kappa \leftrightarrow \mathrm{i} \tau
$$

In the computation of D3-brane action in [17], the metric on the D3-brane is taken to be Euclidean. After the above analytic continuation $\kappa \rightarrow \mathrm{i} \tau, \mathcal{Z}(\tau)$ formally corresponds to a Lorentzian signature D3-brane with an electric field $\tau$. In this picture, the critical value $\tau=1$ can be thought of as the critical electric field for the threshold of holographic Schwinger effect [19]. Appearance of the Lorentzian signature is not surprising since the $\mathrm{SFF}$ captures the chaotic behavior of a system in the real time dynamics.

\footnotetext{
${ }^{3}$ Note that the expectation value of the winding Wilson loop in 2d Yang-Mills theory also has the same expression as $\mathcal{Z}(\tau)$ in $(2.6)[18]$.
} 


\section{Eigenvalue instanton in the spectral form factor}

The large $N$ behavior of $\mathcal{Z}(\tau)$ in (2.6) has been already studied in [13] in the context of 2d Yang-Mills theory on $S^{2}$, where the scaling limit (1.1) corresponds to the 't Hooft limit in the 2d Yang-Mills theory. Here we repeat the same analysis from the viewpoint of eigenvalue instanton in the Gaussian matrix model, in a similar manner as in [20, 21] for the large winding number limit of $1 / 2$ BPS Wilson loop in $4 \mathrm{~d} \mathcal{N}=4$ SYM.

Let us consider the eigenvalue integral representation of the one-point function $\mathcal{Z}(\tau)$ defined in (2.5)

$$
\mathcal{Z}(\tau)=\int \prod_{i=1}^{N} d \mu_{i} e^{-\frac{N}{2} \mu_{i}^{2}} \prod_{1 \leq i<j \leq N}\left(\mu_{i}-\mu_{j}\right)^{2} \sum_{i=1}^{N} e^{2 \mathrm{i} N \tau \mu_{i}} .
$$

Here we have ignored the overall normalization constant for simplicity. Integrating over the $N-1$ eigenvalues which do not couple to the operator insertion $e^{2 \mathrm{i} N \tau \mu_{i}}, \mathcal{Z}(\tau)$ can be written as an integral for a single eigenvalue $x$ which has a non-zero coupling to the operator insertion $e^{2 \mathrm{i} N \tau x}$

$$
\mathcal{Z}(\tau)=\int d x e^{-N V_{\text {eff }}(x)+2 \mathrm{i} N \tau x},
$$

where the effective potential $V_{\text {eff }}(x)$ for the eigenvalue $x$ is defined by integrating out the other $N-1$ eigenvalues

$$
e^{-N V_{\text {eff }}(x)}=e^{-\frac{N}{2} x^{2}} \int \prod_{i=1}^{N-1} d \mu_{i} e^{-\frac{N}{2} \mu_{i}^{2}}\left(x-\mu_{i}\right)^{2} \prod_{1 \leq i<j \leq N-1}\left(\mu_{i}-\mu_{j}\right)^{2} .
$$

In the scaling limit (1.1), the $x$-integral (3.2) can be evaluated by the saddle point approximation. The saddle point $x=x_{*}$ is determined by the condition

$$
V_{\text {eff }}^{\prime}\left(x_{*}\right)=2 \mathrm{i} \tau,
$$

where the prime in $V_{\text {eff }}^{\prime}(x)$ denotes the derivative with respect to $x$. In the large $N$ limit $V_{\text {eff }}^{\prime}(x)$ is written as a sum of the contributions from the Gaussian potential $x^{2} / 2$ and the Coulomb repulsion from the other $N-1$ eigenvalues

$$
V_{\mathrm{eff}}^{\prime}(x)=x-2 \int_{-2}^{2} d y \frac{\rho(y)}{x-y} .
$$

Here $\rho(y)$ denotes the eigenvalue density given by the Wigner semi-circle law

$$
\rho(y)=\frac{1}{2 \pi} \sqrt{4-y^{2}},
$$

and the $N-1$ eigenvalues are distributed along the cut $[-2,2]$ in the large $N$ limit. The integral in (3.5) is easily evaluated as

$$
V_{\text {eff }}^{\prime}(x)=\sqrt{x^{2}-4} .
$$


Using this expression, we find a non-trivial solution for the saddle point equation (3.4)

$$
x_{*}=2 \mathrm{i} v_{*}, \quad v_{*}=\sqrt{\tau^{2}-1} .
$$

Namely, in the scaling limit (1.1) one eigenvalue $x$ is pulled out from the cut $[-2,2]$ to the saddle point $x_{*}=2 \mathrm{i} v_{*}$ on the imaginary axis. This is interpreted as the eigenvalue instanton $[10,11]$ and the instanton action is given by

$$
S_{\mathrm{inst}}(\tau)=\left[V_{\mathrm{eff}}\left(x_{*}\right)-2 \mathrm{i} \tau x_{*}\right]-V_{\mathrm{eff}}\left(x_{0}\right),
$$

where $x_{0}$ is a reference point on the cut $x_{0} \in[-2,2]$. Since $V_{\text {eff }}(x)$ is constant along the cut, one can choose $x_{0}=0$ without loss of generality. Then the instanton action is written as a change of effective potential as we move the eigenvalue along the imaginary axis from $x=0$ to $x=2 \mathrm{i} v_{*}$

$$
S_{\mathrm{inst}}(\tau)=\int_{0}^{x_{*}} d x\left[V_{\mathrm{eff}}^{\prime}(x)-2 \mathrm{i} \tau\right]=4 \int_{0}^{v_{*}} d v\left[-\sqrt{1+v^{2}}+\tau\right] .
$$

In the last equality we have changed the integration variable $x=2 \mathrm{i} v$. One can easily show that (3.10) reproduces the instanton action (2.11) obtained in [13].

Note that in the case of large winding Wilson loop in $\mathcal{N}=4$ SYM one eigenvalue is pulled out of the cut $[-2,2]$ to the saddle point on the real axis $[20,21]$, while in our case the saddle point is on the imaginary axis.

We can repeat the same saddle point analysis for the SFF defined by the matrix integral (2.1). In the scaling limit $(1.1), g_{\text {conn }}(\tau)$ is written as the integral of two eigenvalues $x, y$

$$
g_{\text {conn }}(\tau) \sim \int d x d y(x-y)^{2} e^{-N V_{\text {eff }}(x)-N V_{\text {eff }}(y)+2 \mathrm{i} N \tau(x-y)} .
$$

At the leading order in the large $N$ expansion, the interaction between the two eigenvalues $(x-y)^{2}$ can be neglected and the saddle point equation becomes

$$
V_{\text {eff }}^{\prime}\left(x_{*}\right)=2 \mathrm{i} \tau, \quad V_{\text {eff }}^{\prime}\left(y_{*}\right)=-2 \mathrm{i} \tau .
$$

The solution of this saddle point equation is given by a complex conjugate pair of points on the imaginary axis

$$
x_{*}=2 \mathrm{i} v_{*}, \quad y_{*}=\bar{x}_{*}=-2 \mathrm{i} v_{*},
$$

where $v_{*}$ is defined in (3.8). By evaluating the saddle point value of the effective potential one can see that $g_{\text {conn }}(\tau)$ behaves as the two-instanton correction

$$
g_{\text {conn }}(\tau) \sim e^{-2 N S_{\text {inst }}(\tau)} .
$$

This is consistent with the behavior (2.12) expected from the exact finite $N$ relations (2.4) and (2.8).

Before closing this section, we note that the $\tau$-derivative of instanton action in (3.10) has a simple form. The $\tau$-derivative of the boundary term $v=v_{*}$ in (3.10) vanishes since $\sqrt{1+v_{*}^{2}}=\tau$ and we find

$$
\partial_{\tau} S_{\mathrm{inst}}(\tau)=4 \int_{0}^{v_{*}} d v=4 v_{*}=4 \sqrt{\tau^{2}-1} .
$$

Of course, this agrees with the $\tau$-derivative of $S_{\text {inst }}(\tau)$ in $(2.11)$. 


\section{$4 \quad 1 / N$ expansion around the instanton}

In this section we compute the $1 / N$ corrections to $\partial_{\tau} g_{\text {conn }}(\tau)$ around the instanton in (2.12). This is obtained once we know the $1 / N$ corrections of $\mathcal{Z}(\tau)$ using the relations (2.4) and (2.8). This problem was studied in [13] using the integral representation of Laguerre polynomial. Here we follow the approach in [17] using the differential equation satisfied by $\mathcal{Z}(\tau)$. From the differential equation for the Laguerre polynomial

$$
\left[x \frac{d^{2}}{d x^{2}}+(\alpha+1-x) \frac{d}{d x}+n\right] L_{n}^{\alpha}(x)=0,
$$

one can show that $\mathcal{Z}(\tau)$ obeys

$$
\left[\tau \partial_{\tau}^{2}+3 \partial_{\tau}+16 N^{2} \tau\left(1-\tau^{2}\right)\right] \mathcal{Z}(\tau)=0 .
$$

As discussed in [16], it is convenient to recast this into the equation for the "free energy" $\mathcal{F}(\tau)=-\frac{1}{N} \log \mathcal{Z}(\tau)$

$$
\left(\partial_{\tau} \mathcal{F}\right)^{2}-\frac{1}{N \tau}\left(\tau \partial_{\tau}^{2} \mathcal{F}+3 \partial_{\tau} \mathcal{F}\right)+16\left(1-\tau^{2}\right)=0 .
$$

One can solve this equation order by order in the $1 / N$ expansion

$$
\mathcal{F}(\tau)=\sum_{\ell=0}^{\infty} \frac{1}{N^{\ell}} \mathcal{F}_{\ell}(\tau) .
$$

At the leading order we have

$$
\partial_{\tau} \mathcal{F}_{0}(\tau)=4 \sqrt{\tau^{2}-1},
$$

and from (3.15) the leading term is given by the instanton action in (2.11), as expected

$$
\mathcal{F}_{0}(\tau)=S_{\text {inst }}(\tau) .
$$

One can systematically find the higher order corrections $\mathcal{F}_{\ell}(\tau)$ using the equation (4.4). In this way we find the $1 / N$ expansion of $\mathcal{Z}(\tau)=e^{-N \mathcal{F}(\tau)}$

$$
\mathcal{Z}(\tau)=(-1)^{N-1} \frac{e^{-N S_{\text {inst }}(\tau)}}{\sqrt{32 \pi N} \tau^{\frac{3}{2}}\left(\tau^{2}-1\right)^{\frac{1}{4}}} \sum_{\ell=0}^{\infty} \frac{1}{N^{\ell}} z_{\ell}(\tau),
$$

where the first few terms read

$$
\begin{aligned}
& z_{0}(\tau)=1 \\
& z_{1}(\tau)=-\frac{8 \tau^{4}-12 \tau^{2}+9}{96 \tau\left(\tau^{2}-1\right)^{3 / 2}}, \\
& z_{2}(\tau)=\frac{64 \tau^{8}-192 \tau^{6}+288 \tau^{4}+360 \tau^{2}-135}{18432 \tau^{2}\left(\tau^{2}-1\right)^{3}}, \\
& z_{3}(\tau)=\frac{71168 \tau^{12}-320256 \tau^{10}+554688 \tau^{8}-518400 \tau^{6}-340200 \tau^{4}+170100 \tau^{2}-42525}{26542080 \tau^{3}\left(\tau^{2}-1\right)^{9 / 2}} .
\end{aligned}
$$

Some comments on (4.7) are in order here: 
(i) The overall factor $1 / \sqrt{32 \pi N}$ in (4.7) cannot be determined by the differential equation (4.3) alone. This factor can be obtained by a careful analysis of the Gaussian integral around the saddle point [13].

(ii) As emphasized in [13], the large $N$ limit of $\mathcal{Z}(\tau)$ in (4.7) has an alternating sign $(-1)^{N-1}$, i.e. $\mathcal{Z}(\tau)$ is negative for even $N$ and positive for odd $N$. However, this sign is absent in the disconnected part of the SFF $g_{\text {disc }}(\tau)=\mathcal{Z}(\tau)^{2}$. The connected part $g_{\text {conn }}(\tau)$ does not have this sign either because of the relation $(2.8) .{ }^{4}$

Once we know the $1 / N$ expansion of $\mathcal{Z}(\tau)$ in (4.7), we can easily find the $1 / N$ expansion of $\partial_{\tau} g_{\text {conn }}(\tau)$ using the relations (2.4) and (2.8):

$$
\partial_{\tau} g_{\mathrm{conn}}(\tau)=\frac{e^{-2 N S_{\mathrm{inst}}(\tau)}}{4 \pi \tau\left(\tau^{2}-1\right)} \sum_{\ell=0}^{\infty} \frac{1}{N^{\ell}} g_{\ell}(\tau),
$$

where the first few terms are given by

$$
\begin{aligned}
& g_{0}(\tau)=1, \\
& g_{1}(\tau)=-\frac{8 \tau^{4}+12 \tau^{2}-3}{48 \tau\left(\tau^{2}-1\right)^{3 / 2}}, \\
& g_{2}(\tau)=\frac{64 \tau^{8}+192 \tau^{6}+1248 \tau^{4}-360 \tau^{2}+81}{4608 \tau^{2}\left(\tau^{2}-1\right)^{3}}, \\
& g_{3}(\tau)=\frac{15872 \tau^{12}-94464 \tau^{10}-7488 \tau^{8}-1054080 \tau^{6}+249480 \tau^{4}-127980 \tau^{2}+23085}{3317760 \tau^{3}\left(\tau^{2}-1\right)^{9 / 2}} .
\end{aligned}
$$

We have checked numerically that this expansion is consistent with the exact result of $\partial_{\tau} g_{\text {conn }}(\tau)$ in $(2.7)$.

Apparently, the $1 / N$ expansion of $\partial_{\tau} g_{\text {conn }}(\tau)$ in (4.9) breaks down at $\tau=1$. However, this is just an artifact of the expansion (4.9) and the exact result of $\partial_{\tau} g_{\text {conn }}(\tau)$ in (2.7) is perfectly smooth at $\tau=1$. A similar phenomenon was observed in the Gross-Witten-Wadia model in [22]. As discussed in [22], we can improve the expansion near the critical point using the analogue of the uniform WKB expansion (see [23] for a uniform expansion of the Laguerre polynomial in terms of the Airy function). It would be interesting to study the detail of this uniform expansion.

\section{Comment on the $\beta \neq 0$ case}

In this section, we will briefly comment on the case of non-zero $\beta$. The SFF with non-zero $\beta$ is defined by

$$
g(\beta, \tau)=\left\langle\operatorname{Tr} e^{-(\beta+2 N \mathrm{i} \tau) H} \operatorname{Tr} e^{-(\beta-2 N \mathrm{i} \tau) H}\right\rangle
$$

\footnotetext{
${ }^{4}$ In the partition function of 2d Yang-Mills theory on $S^{2}$, this sign is canceled in the final result after a careful analysis of the extra sign coming from the modular transformation of the partition function [24].
} 
Again, it is decomposed into the disconnected part $g_{\text {disc }}(\beta, \tau)$ and the connected part $g_{\text {conn }}(\beta, \tau)$. The exact form of $\partial_{\tau} g_{\text {conn }}(\beta, \tau)$ was obtained in [9]

$$
\partial_{\tau} g_{\operatorname{conn}}(\beta, \tau)=\frac{2 N^{2}}{\beta} e^{-4 N \tau^{2}+\frac{\beta^{2}}{N}} \operatorname{Im}\left[L_{N}\left(4 N w^{2}\right) L_{N-1}\left(4 N \bar{w}^{2}\right)\right],
$$

with $w, \bar{w}$ being

$$
w=\tau-\mathrm{i} \frac{\beta}{2 N}, \quad \bar{w}=\tau+\mathrm{i} \frac{\beta}{2 N} .
$$

When $\beta \neq 0$, we do not have a simple relation between $g_{\text {disc }}$ and $g_{\text {conn }}$ as in (2.8), and hence the information of the $1 / N$ expansion of the one-point function alone is not enough to find the $1 / N$ expansion of $\partial_{\tau} g_{\text {conn }}(\beta, \tau)$.

We expect that in the scaling limit (1.1) with finite $\beta \sim \mathcal{O}\left(N^{0}\right), \partial_{\tau} g_{\text {conn }}(\beta, \tau)$ is exponentially small in the plateau regime $\tau>1$

$$
\partial_{\tau} g_{\mathrm{conn}}(\beta, \tau) \sim e^{-2 N S_{\mathrm{inst}}(\beta, \tau)} .
$$

In a similar manner as (2.14), we can numerically extract the instanton action $S_{\text {inst }}(\beta, \tau)$ from the exact result of $\partial_{\tau} g_{\text {conn }}(\beta, \tau)$ at finite $N$

$$
S_{\mathrm{inst}}(\beta, \tau) \approx-\frac{1}{2 N} \log \left[\partial_{\tau} g_{\mathrm{conn}}(\beta, \tau)\right], \quad(N \gg 1) .
$$

We observed numerically that $S_{\text {inst }}(\beta, \tau)$ is independent of $\beta$

$$
S_{\text {inst }}(\beta, \tau)=S_{\text {inst }}(\tau),
$$

where the instanton action $S_{\text {inst }}(\tau)$ is given by (2.11). See figure 2 for the plot of (5.5) at $\beta=5$ with $N=500$.

This $\beta$-independence of the instanton action can be understood from the eigenvalue integral considered in (3.11). When $\beta \neq 0,(3.11)$ is modified to

$$
g_{\text {conn }}(\beta, \tau) \sim \int d x d y(x-y)^{2} e^{-N V_{\text {eff }}(x)-N V_{\text {eff }}(y)+2 \mathrm{i} N \tau(x-y)+\beta(x+y)} .
$$

When evaluating this integral in the saddle point approximation, the location of saddle point would be slightly shifted from the $\beta=0$ case in (3.13). However, this shift is subleading in the $1 / N$ expansion as long as $\beta$ is order $N^{0}$ and hence the value of the effective potential at the saddle point does not change at the leading order in the large $N$ expansion. This explains the $\beta$-independence of the instanton action observed numerically in figure 2.

We expect that the $1 / N$ expansion around the instanton for $\beta=0$ in (4.9) is modified when $\beta \neq 0$ and the $\beta$-dependence arises at the subleading order in the $1 / N$ expansion

$$
\partial_{\tau} g_{\mathrm{conn}}(\beta, \tau)=\frac{e^{-2 N S_{\mathrm{inst}}(\tau)}}{4 \pi \tau\left(\tau^{2}-1\right)} \sum_{\ell=0}^{\infty} \frac{1}{N^{\ell}} g_{\ell}(\beta, \tau) .
$$

It would be interesting to study the $\beta$-dependence of the expansion coefficients $g_{\ell}(\beta, \tau)$. We leave this as a future problem. 


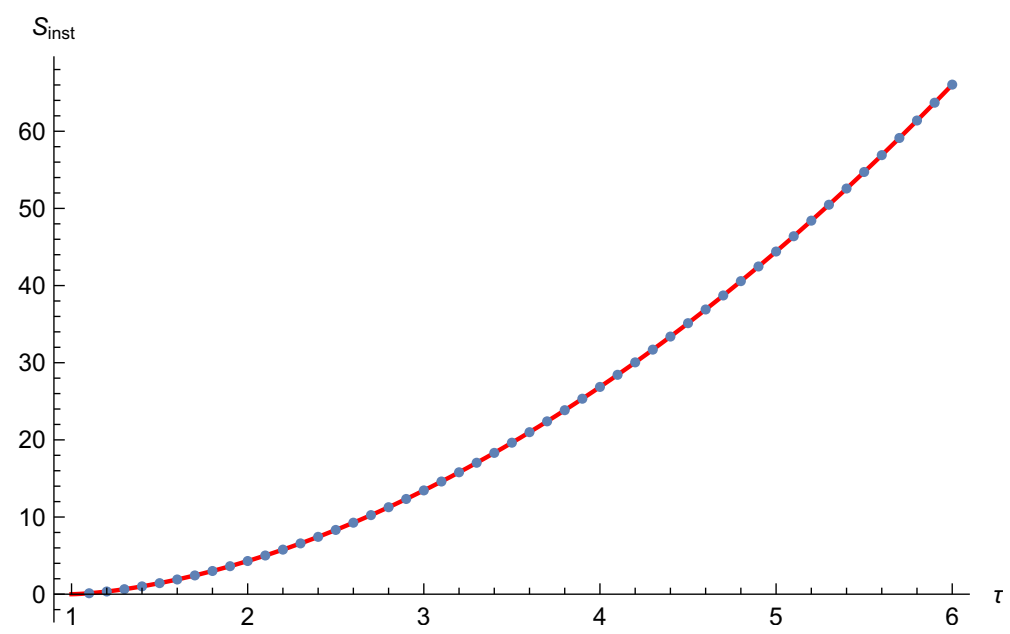

Figure 2. Plot of the instanton action $S_{\text {inst }}(\beta, \tau)$ for $\beta=5$. The dots are the exact values of the right hand side of (5.5) for $\beta=5, N=500$, while the red curve represents the analytic form of $S_{\text {inst }}(\tau)$ at $\beta=0$ in $(2.11)$.

\section{Conclusion and outlook}

In this paper we have studied the exponentially small correction to the connected part of the $\mathrm{SFF}$ in the GUE random matrix model in the plateau regime. We have argued that such a correction comes from the eigenvalue instanton in the matrix model, where two eigenvalues are pulled out from the cut to the complex conjugate pair of saddle points (3.13) on the imaginary axis. We found the analytic form of the instanton action (2.11) as a function of time and demonstrated that the $1 / N$ corrections around the instanton configuration can be systematically obtained from the differential equation (4.3) and the non-trivial relation (2.8) between the connected and the disconnected part of the SFF.

At the technical level, the non-perturbative corrections in the SFF in the GUE matrix model is formally equivalent to the same problem in the $2 \mathrm{~d}$ Yang-Mills theory on $S^{2}$. In this correspondence, the ramp-plateau transition in the SFF is related to the Douglas-Kazakov phase transition in the 2d Yang-Mills theory on $S^{2}$. Also, we have seen that this problem is related to the large winding number limit of the $1 / 2$ BPS Wilson loop in $4 \mathrm{~d} \mathcal{N}=4$ SYM after an analytic continuation (2.18).

The study in this paper is strongly motivated by the recent discussion of a possible interpretation of the plateau in the SFF of the SYK model $[2,6]$. It was advocated in $[2,6]$ that the plateau in the SYK model is closely related to the non-perturbative effect in the $1 / N$ expansion, which is identified with the effect of D-branes in the holographic dual string theory of SYK model, dubbed the "JT string" in [6]. In this paper, we have seen that this picture is indeed realized in the simple example of the Gaussian matrix model where the role of D-brane is played by the eigenvalue instanton in the matrix model. In the analogy with the large winding Wilson loop in $\mathcal{N}=4 \mathrm{SYM}$, the appearance of a complex conjugate pair of saddle points can be thought of as a pair creation of D-branes by the Schwinger effect. It would be very interesting to see how such non-perturbative effects arise in the 
case of the SYK model. Perhaps, one can use the relation between the spectrum of SYK model and the $q$-Hermite polynomials discussed in [25].

The non-perturbative correction to the SFF is an interesting problem in its own right, regardless of the connection to the SYK model and quantum gravity. In this paper, we have seen that the ramp-plateau transition of the SFF of GUE matrix model can be thought of as a kind of phase transition induced by the eigenvalue instantons. It would be interesting to study the non-perturbative correction in other ensembles, such as GOE and GSE, and see if the above picture of phase transition applies to other models as well. The saddle point analysis for the SFF of GOE and GSE might be tractable, as we did for GUE in section 3. Also, using the known result of $1 / 2$ BPS Wilson loops in $4 \mathrm{~d} \mathcal{N}=4 \mathrm{SYM}$ with gauge group $\mathrm{SO}(N)$ and $\mathrm{Sp}(N)$ [26], it would be possible to write down the exact $\mathrm{SFF}$ of GOE and GSE at finite $N$ and study its large $N$ limit. We leave this as an interesting future problem.

\section{Acknowledgments}

I would like to thank Shinji Hirano, Tadakatsu Sakai, Yuki Sato and Masaki Shigmeori for discussion. This work was supported in part by JSPS KAKENHI Grant Number 16K05316.

Open Access. This article is distributed under the terms of the Creative Commons Attribution License (CC-BY 4.0), which permits any use, distribution and reproduction in any medium, provided the original author(s) and source are credited.

\section{References}

[1] L. Leviandier, M. Lombardi, R. Jost and J.P. Pique, Fourier Transform: A Tool to Measure Statistical Level Properties in Very Complex Spectra, Phys. Rev. Lett. 56 (1986) 2449.

[2] J.S. Cotler et al., Black Holes and Random Matrices, JHEP 05 (2017) 118 [Erratum ibid. 09 (2018) 002] [arXiv: 1611.04650] [INSPIRE].

[3] A. Kitaev, A simple model of quantum holography, talks at KITP (2015).

[4] S. Sachdev and J. Ye, Gapless spin fluid ground state in a random, quantum Heisenberg magnet, Phys. Rev. Lett. 70 (1993) 3339 [cond-mat/9212030] [INSPIRE].

[5] J. Maldacena and D. Stanford, Remarks on the Sachdev-Ye-Kitaev model, Phys. Rev. D 94 (2016) 106002 [arXiv:1604.07818] [INSPIRE].

[6] P. Saad, S.H. Shenker and D. Stanford, A semiclassical ramp in SYK and in gravity, arXiv: 1806.06840 [INSPIRE].

[7] E. Brézin and S. Hikami, Spectral form factor in a random matrix theory, Phys. Rev. E 55 (1997) 4067 [cond-mat/9608116].

[8] J. Liu, Spectral form factors and late time quantum chaos, Phys. Rev. D 98 (2018) 086026 [arXiv: 1806.05316] [INSPIRE].

[9] K. Okuyama, Spectral form factor and semi-circle law in the time direction, JHEP 02 (2019) 161 [arXiv: 1811.09988] [INSPIRE]. 
[10] F. David, Phases of the large $N$ matrix model and nonperturbative effects in 2-D gravity, Nucl. Phys. B 348 (1991) 507 [INSPIRE].

[11] F. David, Nonperturbative effects in matrix models and vacua of two-dimensional gravity, Phys. Lett. B 302 (1993) 403 [hep-th/9212106] [INSPIRE].

[12] A.V. Andreev and B.L. Altshuler, Spectral Statistics Beyond Random Matrix Theory, Phys. Rev. Lett. 75 (1995) 902 [cond-mat/9503141].

[13] D.J. Gross and A. Matytsin, Instanton induced large $N$ phase transitions in two-dimensional and four-dimensional QCD, Nucl. Phys. B 429 (1994) 50 [hep-th/9404004] [InSPIRE].

[14] M.R. Douglas and V.A. Kazakov, Large $N$ phase transition in continuum $Q C D$ in two-dimensions, Phys. Lett. B 319 (1993) 219 [hep-th/9305047] [INSPIRE].

[15] M. Mariño, Nonperturbative effects and nonperturbative definitions in matrix models and topological strings, JHEP 12 (2008) 114 [arXiv:0805.3033] [INSPIRE].

[16] N. Drukker and D.J. Gross, An Exact prediction of $N=4$ SUSYM theory for string theory, J. Math. Phys. 42 (2001) 2896 [hep-th/0010274] [INSPIRE].

[17] N. Drukker and B. Fiol, All-genus calculation of Wilson loops using D-branes, JHEP 02 (2005) 010 [hep-th/0501109] [INSPIRE].

[18] D.J. Gross and A. Matytsin, Some properties of large $N$ two-dimensional Yang-Mills theory, Nucl. Phys. B 437 (1995) 541 [hep-th/9410054] [INSPIRE].

[19] G.W. Semenoff and K. Zarembo, Holographic Schwinger Effect, Phys. Rev. Lett. 107 (2011) 171601 [arXiv:1109.2920] [INSPIRE].

[20] S. Kawamoto, T. Kuroki and A. Miwa, Boundary condition for D-brane from Wilson loop and gravitational interpretation of eigenvalue in matrix model in AdS/CFT correspondence, Phys. Rev. D 79 (2009) 126010 [arXiv:0812.4229] [INSPIRE].

[21] S.A. Hartnoll and S.P. Kumar, Higher rank Wilson loops from a matrix model, JHEP 08 (2006) 026 [hep-th/0605027] [INSPIRE].

[22] A. Ahmed and G.V. Dunne, Transmutation of a Trans-series: The Gross-Witten-Wadia Phase Transition, JHEP 11 (2017) 054 [arXiv: 1710.01812] [INSPIRE].

[23] Asymptotic Approximations, Digital Library of Mathematical Functions, section 18.15, https://dlmf.nist.gov/18.15.

[24] K. Okuyama, Phase diagram of q-deformed Yang-Mills theory on $S^{2}$ at non-zero $\theta$-angle, JHEP 04 (2018) 059 [arXiv: 1801.08236] [INSPIRE].

[25] A.M. García-García and J.J.M. Verbaarschot, Analytical Spectral Density of the Sachdev-Ye-Kitaev Model at finite N, Phys. Rev. D 96 (2017) 066012 [arXiv:1701.06593] [INSPIRE].

[26] B. Fiol, B. Garolera and G. Torrents, Exact probes of orientifolds, JHEP 09 (2014) 169 [arXiv: 1406.5129] [INSPIRE]. 